FRI0475

\section{LUNG DAMAGE IN PATIENTS WITH MICROSCOPIC} POLYANGIITIS

E. Shchegoleva ${ }^{1}$, A. Zykova ${ }^{2}$, N. Bulanov ${ }^{1}$, A. Meshkov ${ }^{1}$, P. Novikov ${ }^{1}$, S. Moiseev'. '1 Department of internal diseases, Sechenov First Moscow State Medical University; ${ }^{2}$ Department of internal diseases, Lomonosov Moscow State University, Moscow, Russian Federation

Objectives: We evaluated the frequency of clinical and CT features of lung damage in patients with microscopic polyangiitis (MPA).

Methods: We enrolled 97 patients with MPA, that was diagnosed according to $\mathrm{CHCC2012}$. The median age at disease onset was 50.7 $\pm 16.6(\mathrm{M} \pm \mathrm{SEM})$ years, the median duration of follow-up was $47.6 \pm 47.5(\mathrm{M} \pm \mathrm{SEM})$ months. $64(66.0 \%)$ patients were ANCA-MPO-positive, 24 patients (24.7\%) were ANCA-PR-3-positive, and $9(9.3 \%)$ patients had undifferentiated ANCA. Diffuse alveolar haemorrhage (DAH) was diagnosed by the presence of dyspnea, hemoptysis, anaemia and pulmonary infiltrates on chest CT.

Results: Lung damage was diagnosed in 77 (79.4\%) patients. 43 (55.8\%) patients had pulmonary damage at the disease onset, while $34(44.2 \%)$ patients developed signs of lung involvement within 8.0 $\pm 4.1(\mathrm{M} \pm \mathrm{SEM})$ months. At base line, the median pulmonary BVAS was $4 .^{2 ; 6}$ The interstitial changes occurred in more than half of cases at the onset of the disease. The most frequent CT-patterns included pulmonary infiltrates $(n=49)$ and ground-glass opacity $(n=39)$ (table 1). $\mathrm{DAH}$ developed in 30 (30.9\%) patients, among them $15(15.5 \%)$ had DAH at the onset of the disease.

The pulmonary fibrosis was the most common CT-pattern at the end of follow-up (52 patients). Notably, interstitial damages at the onset of disease were associated with the development of fibrotic changes (OR=4.7, 95\% $\mathrm{Cl} 1.7-12.9)$ and bronchiectasis $(\mathrm{OR}=9.8,95 \% \mathrm{Cl} 1.2-78.3)$ at the end of follow-up. The median of pulmonary VDI was $1(0 ; 4)$ at the end of the follow up.

PR-3-positive group had higher occurrence of consolidations at the end of the follow-up as compared to patients with anti-MPO-antibodies (53.8\% versus $16.0 \%$, $\mathrm{p}=0.023$ )

\begin{tabular}{lcc}
\hline Abnormality & \multicolumn{2}{c}{$\mathrm{N}(\%)$ of cases } \\
\cline { 2 - 3 } & At baseline $(\mathrm{n}=77)$ & At the end of follow-up $(\mathrm{n}=47)$ \\
\hline Interstitial damage & $68(70.1 \%)$ & $18(18.6 \%)$ \\
Infiltrates & $49(50.5 \%)$ & $0(0 \%)$ \\
Ground-glass opacity & $39(40.2 \%)$ & $16(16.5 \%)$ \\
Consolidation & $5(5.2 \%)$ & $8(8.2 \%)$ \\
Bronchial wall thickening & $10(10.3 \%)$ & $18(18.6 \%)$ \\
Bronchiectasis & $4(4.1 \%)$ & $18(18.6 \%)$ \\
Bronchiolitis & $3(3.1 \%)$ & $5(5.2 \%)$ \\
Pleural lesions & $11(11.3 \%)$ & $5(5.2 \%)$ \\
Pleural effusion & $12(12.4 \%)$ & $0(0 \%)$ \\
Pleural thickening & $3(3.1 \%)$ & $5(5.2 \%)$ \\
Pulmonary fibrosis & $14(14.1 \%)$ & $52(53.6 \%)$ \\
Honeycombing & $0(0.0 \%)$ & $4(4.1 \%)$ \\
Atelectasis & $3(3.1 \%)$ & $8(8.2 \%)$ \\
Emphysema & $0(0.0 \%)$ & $13(13.4 \%)$ \\
Pulmonary hypertension & $0(0.0 \%)$ & $7(7.2 \%)$ \\
\hline
\end{tabular}

The pulmonary fibrosis was the most common CT-pattern at the end of follow-up (52 patients). Notably, interstitial damages at the onset of disease were associated with the development of fibrotic changes (OR=4.7, 95\% $\mathrm{Cl} 1.7-12.9)$ and bronchiectasis $(\mathrm{OR}=9.8,95 \% \mathrm{Cl} 1.2-78.3)$ at the end of follow-up. The median of pulmonary VDI was $1(0 ; 4)$ at the end of the follow up.

PR-3-positive group had higher occurrence of consolidations at the end of the follow-up as compared to patients with anti-MPO-antibodies (53.8\% versus $16.0 \%$, $\mathrm{p}=0.023$ )

Conclusions: In patients with MPA, the CT signs of interstitial damage were usually reversible. However, they predicted a higher incidence of lung fibrosis and bronchiectasis at the end of follow-up. DAH occured in one third of patients with MPA

Disclosure of Interest: None declared

DOI: 10.1136/annrheumdis-2018-eular.5715

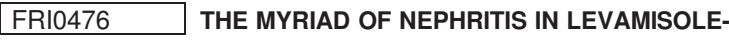 ADULTERATED COCAINE VASCULOPATHY}

C.H. Munoz ${ }^{1,2,3}$, S. Herrera-Uribe ${ }^{4}$, Á. Arbeláez-Cortés ${ }^{5}$, D. Jaramillo-Arroyave $e^{2,3}$, L.A. González-Naranjo ${ }^{2}$, G. Vásquez-Duque ${ }^{2}$, M. Restrepo-Escobar ${ }^{2}$, J. Hernández-Zapata ${ }^{2}$,L.F. Arias-Restrepo ${ }^{6}$, A.L. Vanegas-García ${ }^{1} .{ }^{1}$ Hospital Universitario San Vicente Fundación; ${ }^{2}$ Grupo de Reumatología, Departamento de Medicina Interna Universidad de Antioquia; ${ }^{3}$ IPS Universitaria Clínica León XIII; ${ }^{4}$ Hospital General de Medellín, Medellin; ${ }^{5}$ Centro Médico Imbanaco de Cali, Cali, ${ }^{6}$ Departamento de Patología, Universidad de Antioquia, Medellin, Colombia

Background: Up to $88 \%$ of cocaine is tainted with levamisole, an anthelmintic withdrawn from the market due to toxicity. Since 2010 levamisole-adulterated cocaine vasculopathy (LACIV) patients, characterised by retiform purpura, ear necrosis, multisystemic compromise and positivity for multiple autoantibodies, have been reported. Renal involvement is the most serious and heterogeneous manifestation.

Objectives: To describe the renal involvement of patients with LACIV.

Methods: We describe the renal manifestations of 30 patients with LACIV evaluated from December 2010 to May 2017.

Results: All patients were mestizo, median age of 31 (IQR 27-38), male:female ratio 5:1, time from symptoms to diagnosis 12 months (IQR 6-24). Nephritis found in $57 \%$, creatinine elevation in $40 \%$, median $1.85 \mathrm{mg} / \mathrm{dl}$ (IQR $1.2-4.0$ ), $70 \%$ had proteinuria, median 3184 mg/day (IQR 552-5100), 58\% in nephrotic-range; 88\% had hematuria and $41 \%$ pyuria and cilindruria. Biopsy was performed in 7 patients $(41 \%)$, showing immune complex mediated extracapillary glomerulonephritis (29\%), membranous glomerulonephritis $(29 \%)$, pauci-immune proliferative glomerulonephritis (14\%), focal and segmental glomerulosclerosis (14\%) and C3 mediated extracapillary glomerulonephritis (14\%) (image). Three patients $(10 \%)$ developed end-stage kidney disease.

Image: A. Membranous glomerulonephritis: HE: glomerular thickened capillary walls, mild mesangial cellularity increase. B. GMS: capillary wall thickening and spikes (arrow). C. DIF: strong and diffuse IgG deposits on capillary walls. D. Immune complex mediated Extracapillary Focal Necrotizing Glomerulonephritis: HE: endocapillary proliferation, epithelial crescent (arrow). E. GMS capillary wall rupture next to the crescent (arrow). F. DIF: Mesangial strong and diffuse IgG deposits. G. C3 mediated extracapillary glomerulonephritis: HE: glomerular capillary narrowing, epithelial crescent (arrow). H. GMS: normal capillary networks, epithelial crescent (arrow). I. DIF: C3 strong and diffuse positivity J. Pauci-immune proliferative glomerulonephritis: PAS: epithelial crescents (arrows and asterisk). K. Trichrome: epithelial crescent (arrow). L. DIF for C3: weak deposits on capillary tuft. Non-immune complexes. M. Focal and segmental glomerulosclerosis: HE: segmental sclerosing lesions (arrow). N. PAS: seg mental sclerosing lesions (asterisks). O. DIF for IgM: nonspecific positivity on a sclerotic segment.

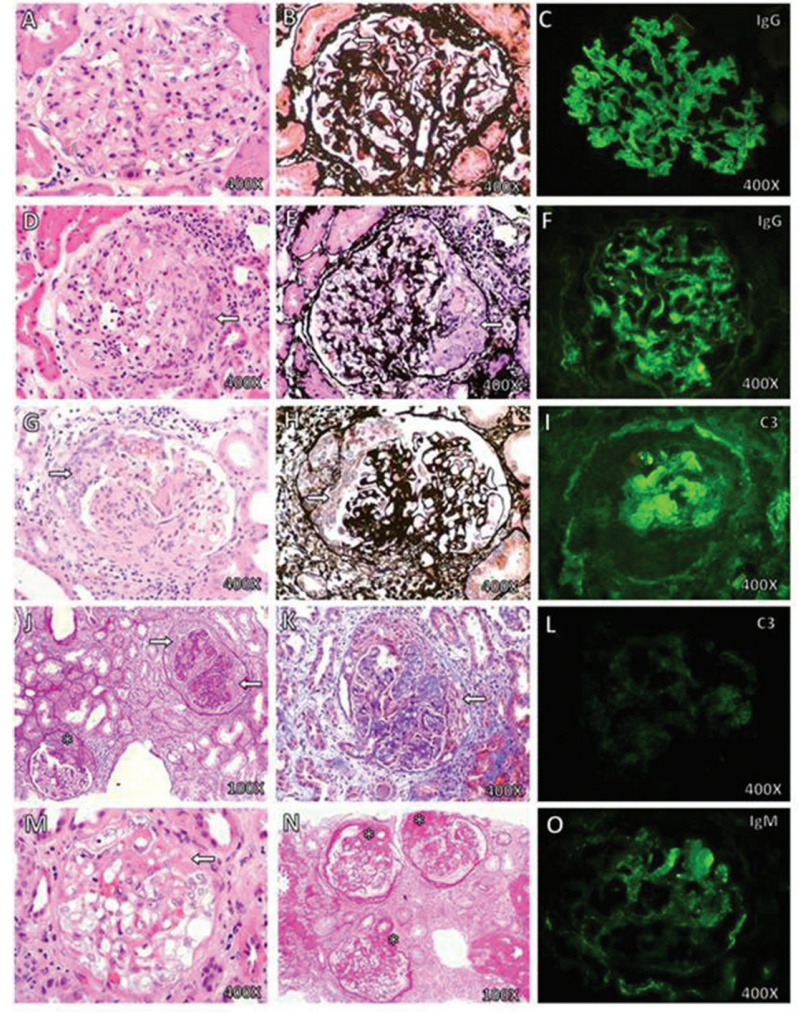

Conclusions: Although skin manifestations are the most characteristic and prevalent features in LIVEN, renal involvement is frequent, clinically and histologically heterogeneous, and potentially serious. The great heterogeneity on the histopathological findings suggests the participation of different physio-pathological mechanisms, establishing renal biopsy as necessary to identify the type of nephropathy and thus, optimal guidance of therapy.

\section{REFERENCES}

[1] Collister D, et al. Am J Nephrol. 2017;45(3):209-16.

[2] Carlson AQ, et al. Medicine (Baltimore) 2014 Oct;93(17):290-7. 